\title{
UHPLC - Uma abordagem atual: desenvolvimentos e desafios recentes
}

\author{
Liane Maldaner, Isabel Cristina Sales Fontes Jardim* \\ Instituto de Química, Universidade Estadual de Campinas - UNICAMP, Cep 13083-970, Campinas, SP, Brasil \\ e-mail: icsfj@iqm.unicamp.br
}

Resumo

A cromatografia líquida de ultra eficiência (UHPLC) desenvolveu-se com a introdução das partículas de fases estacionárias (FE) porosas $\leq 2 \mu \mathrm{m}$, juntamente com a busca contínua por análises mais rápidas e eficientes, e fundamenta-se nos mesmos princípios de separação da cromatografia líquida de alta eficiência (HPLC). Desde a sua introdução em 2004, a UHPLC vem ganhando espaço em todas as áreas de aplicação da HPLC em decorrência de suas vantagens e, desta forma, vem sendo alvo constante de novas pesquisas, principalmente no que diz respeito a novas $\mathrm{FE}$ e melhorias nos equipamentos. Os principais desafios no emprego da UHPLC em comparação com a HPLC já foram identificados e juntamente com os desenvolvimentos recentes serão abordados neste artigo.

Palavras-chave

UHPLC; fases estacionárias; instrumentação; UHPLC $\times$ HPLC.

\section{UHPLC - The present situation: developments and recent challenges}

\section{Abstract}

Ultra-high performance liquid chromatography (UHPLC) emerged with the advent of sub-2 $\mu \mathrm{m}$ porous particles catalyzed by the continuous search for faster and more efficient analyses, and retains the same separation principles as high performance liquid chromatography (HPLC). Since its introduction in 2004, UHPLC has grown in all HPLC separation areas as a consequence of its advantages and, thus, has been a driving force for new research, especially in terms of novel stationary phases and improved equipment. The main challenges for using UHPLC, in comparison with HPLC, have already been identified and will be discussed in this paper together with recent developments in both stationary phases and instrumentation.

Keywords

UHPLC; stationary phases; instrumentation; UHPLC $\times$ HPLC. 


\section{Introdução}

A cromatografia líquida de alta eficiência (HPLC) é uma técnica de separação bem estabelecida e é empregada nas mais diversas áreas, entre elas, química, forense, toxicológica, clínica e ambiental, para solucionar inúmeros problemas analíticos. Durante os últimos anos, muitas melhorias vêm sendo incorporadas a esta técnica, como desenvolvimentos de novas fases estacionárias (FE) e suportes cromatográficos, avanços na instrumentação, entre outros, permitindo que análises mais rápidas e mais eficientes sejam alcançadas, corroborando com a necessidade atual de aumentar o número de análises e o rendimento e reduzir os custos.

A cromatografia líquida de ultra eficiência (UHPLC) ${ }^{[1-7]}$ (UHPLC - ultra-high pressure liquid chromatography ou ultra-high performance liquid chromatography) desenvolveu-se a partir da introdução das partículas de FE porosas $\leq 2 \mu \mathrm{m}$, em resposta à busca contínua por análises mais rápidas e eficientes. A UHPLC fundamenta-se nos mesmos princípios de separação da HPLC, tendo como principais diferenças as colunas cromatográficas empregadas que são de dimensões reduzidas (5-10 $\mathrm{cm}$ de comprimento e diâmetros internos de 1-2,1 mm), recheadas com partículas de $\mathrm{FE} \leq 2 \mu \mathrm{m}$, as quais, juntamente com as altas velocidades lineares de fase móvel (FM) aumentam a resolução e a detectabilidade, diminuem o tempo das análises, porém geram um aumento significativo na pressão cromatográfica. Em vista disso, um equipamento adequado, capaz de operar a altas pressões, acima de 1000 bar $(\sim 15000$ psi), é empregado para extrair um desempenho cromatográfico máximo dessa técnica.

Desde a introdução do primeiro equipamento de UHPLC pela Waters Corporation em 2004, denominado de ACQUITY UPLC ${ }^{\circledast}$ System, que permitiu que a técnica fosse empregada além do meio acadêmico e avaliada por usuários diversos, até os dias atuais, a UHPLC vem ganhando espaço em todas as áreas de aplicação da HPLC, em decorrência de suas principais vantagens: diminuição considerável no tempo de análise, melhor resolução e detectabilidade, economia de fase estacionária e fase móvel, pequeno volume de amostra, facilidade de transferência de um método desenvolvido por HPLC para UHPLC, grande variedade de colunas e equipamentos disponíveis e menor geração de resíduos atendendo, desta forma, à Química Verde.

Em vista deste rápido crescimento e da expansão do emprego da UHPLC nas análises de rotina, esta técnica vem sendo alvo constante de novas pesquisas, principalmente no que diz respeito a novas FE e suportes cromatográficos e melhorias nos equipamentos. Os principais desafios no uso da UHPLC em comparação com a HPLC já foram identificados e, juntamente com os desenvolvimentos recentes, serão abordados neste artigo.

\section{Desenvolvimentos recentes}

O desenvolvimento da UHPLC se deu em virtude da introdução das partículas de FE porosas $\leq 2 \mu \mathrm{m}$ que, associadas às colunas cromatográficas com dimensões reduzidas e às altas velocidades lineares de FM, permitiram que análises mais rápidas fossem possíveis, sem o comprometimento da eficiência e da resolução cromatográfica. Entretanto, a grande aceitação da UHPLC nos mais diversos campos de aplicação impulsionou as pesquisas em busca de novos materiais, suportes e/ou FE e novas estratégias de uso das colunas e dos equipamentos para ancorar o crescimento e também para oferecer novas alternativas aos desafios decorrentes da expansão e da ampla variedade de áreas de aplicação. 


\subsection{Colunas de UHPLC: novos suportes e fases estacionárias}

\subsubsection{Colunas recheadas com partículas porosas $\leq 2 \mu \mathrm{m}$}

As primeiras colunas recheadas com partículas de $\mathrm{FE} \leq 2 \mu \mathrm{m}$ disponíveis comercialmente foram as denominadas ACQUITY BEH (BEH - ethylene bridged hybrid) (baseadas em sílica híbrida de segunda geração de $1,7 \mu \mathrm{m}$, a qual possui pontes de etano inseridas na sua estrutura), introduzidas em 2004 pela Waters Corporation. Hoje, 8 anos após o surgimento da técnica, mais de 30 fabricantes estão comercializando colunas de UHPLC recheadas com FE de tamanhos de partículas que variam de 1,5 a 2,0 $\mu \mathrm{m}$, preparadas a partir de suportes de sílica ou de sílica híbrida e modificadas com diferentes grupos químicos como: $\mathrm{C} 18, \mathrm{C} 8$, fenil, ciano e grupos polares embutidos ${ }^{[2,4,5,8,9]}$.

Além destas FE, muitas outras estão sendo desenvolvidas e empregadas em condições de UHPLC, como as FE baseadas em suporte de sílica de elevada resistência (HSS) (HSS - high strenght silica) e suportes híbridos com a superfície carregada $(\mathrm{CSH})$ ( $\mathrm{CSH}$ - charged surface hybrid), HILIC (HILIC - hydrophilic interaction chromatography), fluoradas, de modo misto, entre outras, como alternativas para as separações cromatográficas mais complexas não alcançadas com as FE tradicionais de fase reversa (FR).

$\mathrm{O}$ suporte cromatográfico designado de HSS foi desenvolvido pela Waters Corporation como alternativa às partículas de sílica híbrida de segunda geração $(\mathrm{BEH})$ de $1,7 \mu \mathrm{m}^{[10]}$. Este suporte cromatográfico é constituído de $100 \%$ de sílica, de tamanho de partícula de 1,8 $\mu \mathrm{m}$ e, segundo o fabricante, possui uma morfologia que é capaz de aumentar o tempo de vida útil, gerar maiores eficiências e proporcionar alta resistência mecânica, podendo ser empregado em pres- sões de até 1034 bar ( 15000 psi). Encontram-se disponíveis comercialmente colunas HSS C18, ciano e fluorfenil.

Mais recentemente, a Waters Corporation lançou as partículas de sílica híbrida de terceira geração, que consistem de partículas de sílica híbrida (partículas BEH de 1,7 $\mu \mathrm{m}$ ) incorporadas com uma pequena quantidade de carga na superfície $^{[11]}$. As principais vantagens que estas partículas apresentam são a melhora na capacidade de amostra e na simetria de pico de compostos básicos quando são empregadas FM com baixa força iônica (meio ácido). Essas partículas são comercializadas pela Waters com a denominação de $\mathrm{CSH}$ e encontram-se disponíveis comercialmente colunas $\mathrm{C} 18$, fenil-hexil e fluorfenil.

As FE denominadas de HILIC são empregadas em cromatografia de interação hidrofílica (HILIC - hydrophilic interaction chromatography), que é uma modalidade de separação na qual é empregada uma FE polar e uma FM menos polar, sendo, desta forma, muito propícia para análise de compostos polares e iônicos ${ }^{[5,12]}$. As principais vantagens da HILIC em relação às separações por fase reversa, que fizeram com que elas também fossem redimensionadas para serem empregadas em UHPLC são: a ordem de eluição dos compostos em HILIC é mais ou menos oposta à ordem de eluição em FR, o que indica que a HILIC retém melhor os compostos que são problemáticos de serem analisados em FR; alta detectabilidade nas análises com detecção por espectrometria de massas (EM) devido à alta porcentagem de solvente orgânico polar empregada nas FM para HILIC e separações mais rápidas, devido a menor viscosidade da FM.

Uma alternativa para aumentar a seletividade das separações por FR é a modificação ou introdução de novos grupos químicos nas cadeias da $\mathrm{FE}^{[5,12]}$. Dentro deste contexto, as FE que vêm ganhando destaque são as $\mathrm{FE}$ fluoradas, 
por apresentarem uma seletividade única proveniente das propriedades específicas das ligações C-F que promovem um aumento no caráter dipolar da FE, o que intensifica a interação com compostos polares, e, dessa forma, as separações não ocorrem por um mecanismo de interação simples de FR. Entre as FE fluoradas, podem ser destacados dois tipos, aquelas que possuem os átomos de flúor ligados à cadeia alquila e aquelas que possuem os átomos de flúor ligados a grupos fenil. Estas últimas, além de possuírem a seletividade influenciada pelas propriedades específicas das ligações C-F, são também influenciadas pelas interações $\pi-\pi$, provenientes dos grupos fenil. Em vista disso, colunas recheadas com FE fluoradas com dimensões apropriadas para UHPLC vêm sendo comercializadas por diversos fabricantes e exploradas em diferentes campos de aplicação.

\subsubsection{Colunas recheadas com partículas superficialmente porosas sub-3 $\mu \mathrm{m}$ e sub- $2 \mu \mathrm{m}$}

Conforme descrito nas seções anteriores, tanto a introdução da UHPLC quanto a expansão da técnica se deu com o advento das partículas de FE porosas $\leq 2 \mu \mathrm{m}$. Entretanto, atualmente, praticamente todos os novos desenvolvimentos e/ou artifícios em torno de novas FE ou de novas estratégias de trabalho podem também ser transferidas/aplicadas às separações realizadas por UHPLC.

Um exemplo deste fato são as partículas de sílica superficialmente porosas (SPP) (SPP - superficially porous particles), que são compostas por um núcleo sólido revestido por uma fina camada de sílica porosa, que foram desenvolvidas como uma alternativa às partículas de FE porosas $\leq 2 \mu \mathrm{m}$, por fornecerem análises rápidas e com eficiências similares, porém sem um aumento significativo da pressão cromatográfica, sendo, desta forma, compatíveis com os sistemas convencionais de $\operatorname{HPLC}^{[2,5,12,13-17]}$. Estas vantagens são decorrentes das características destas partículas, que são produzidas com uma estreita faixa de distribuição de tamanho e, desta forma, permitem maior densidade de recheio das colunas cromatográficas, e, devido à fina camada porosa, oferecem uma baixa resistência à transferência de massa, possibilitando o emprego de maiores velocidades lineares de FM sem perdas significativas na eficiência.

Estas partículas são comercializadas por diferentes fabricantes, como: com a denominação de $\mathrm{Halo}^{\mathrm{TM}}$, pela Advanced Material Technologies; Kinetex ${ }^{\mathrm{TM}}$, pela Phenomenex; Ascentis $^{\mathrm{TM}}$ Express, pela Supelco; Poroshell 120, pela Agilent Technologies; Nucleoshell ${ }^{\circledR}$, pela Macherey-Nagel; Sunshell, pela Biotech (patenteado pela Chromanik Technologies Inc.); e Accucore, pela Thermo Scientific, com tamanhos de partículas $\leq 3 \mu \mathrm{m}$ (sub-3 $\mu \mathrm{m})$ e $\leq 2 \mu \mathrm{m}$ (sub-2 $\mu \mathrm{m}$ ), sendo estas últimas apenas comercializadas pela Phenomenex. Partículas superficialmente porosas $\leq 2 \mu \mathrm{m}$ também vêm sendo desenvolvidas pela Glantreo Ltd, Ireland, com a denominação de Eiroshell $^{\mathrm{TM}}$, porém ainda não se encontram disponíveis comercialmente ${ }^{[13-15]}$. As partículas sub-3 $\mu \mathrm{m}$ são resistentes a pressões de até 600 bar ( 9000 psi) e comercializadas em colunas com diâmetros internos de 4,6, 3,0 e 2,1 mm; e as partículas sub- $2 \mu \mathrm{m}$ são resistentes a pressões de até 1000 bar ( 15000 psi) e comercializadas em colunas com diâmetros internos de 3,0 e 2,1 mm. Encontram-se disponíveis FE preparadas a partir das partículas superficialmente porosas, sub-3 $\mu \mathrm{m}$ e sub- $2 \mu \mathrm{m}$, com diferentes grupos químicos como: $\mathrm{C} 18, \mathrm{C} 8$, fenil, flúor, grupos polares embutidos, HILIC, entre outros.

Embora sejam, na maioria das vezes, vistas como uma alternativa ao emprego da UHPLC e, consequentemente, das partículas de FE porosas $\leq 2 \mu \mathrm{m}$, quando se trata de cromatografia 
rápida, estas partículas superficialmente porosas, sub-3 $\mu \mathrm{m}$ ou sub- $2 \mu \mathrm{m}$, podem ser empregadas em conjunto com a UHPLC para que análises mais seletivas e/ou com melhor ou diferente desempenho cromatográfico sejam alcançadas. Estudos comparativos a respeito das vantagens do emprego das partículas superficialmente porosas associadas ao UHPLC em relação às partículas porosas $\leq 2 \mu \mathrm{m}$ ainda não foram concluídos, mas indicam que, em termos de eficiência, as partículas superficialmente porosas parecem ser mais vantajosas.

\subsection{Condições de operação das colunas: emprego de temperatura}

Uma alternativa para melhorar o desempenho cromatográfico bem como tornar as análises por cromatografia líquida mais rápidas é através do emprego de altas temperaturas $\left(60<\mathrm{T}<200{ }^{\circ} \mathrm{C}\right)^{[2,4,5,12,18]}$. O emprego de temperatura mais elevada reduz a viscosidade da FM e, desta forma, a resistência à transferência de massa também diminui, permitindo o uso de maiores vazões de FM sem aumento na pressão cromatográfica, e, consequentemente, análises mais rápidas são alcançadas. Além disso, o emprego da temperatura pode melhorar a eficiência e a resolução cromatográfica.

O emprego da temperatura pode ser utilizado nas separações por UHPLC, assim como nas análises por HPLC, possuindo as mesmas vantagens e limitações. Entretanto, a expansão do emprego da temperatura nas separações cromatográficas fora do meio acadêmico ainda é lenta, devido ao número limitado de $\mathrm{FE}$ que são estáveis em temperaturas acima de $60{ }^{\circ} \mathrm{C}$, a necessidade de modificação do equipamento para controlar adequadamente a temperatura da FM e a possibilidade de degradação dos compostos a serem analisados.
A maioria das FE baseadas em sílica possui estabilidade térmica até $60{ }^{\circ} \mathrm{C}$, independentemente se for indicada para uso em HPLC ou em UHPLC. Entre as FE que vêm sendo desenvolvidas para serem empregadas em altas temperaturas $\left(\geq 150^{\circ} \mathrm{C}\right)$, como as poliméricas, as baseadas em zircônia ou em carbono grafitizado, apenas se encontram disponíveis comercialmente, com dimensões de UHPLC e recheadas com partículas $\leq 2 \mu \mathrm{m}$, colunas baseadas em zircônia, comercializadas pela ZirCrom $^{\oplus}{ }^{[19]}$, a Zicrom $^{\circledR}$-PBD e a Zircrom $^{\oplus}$-PHASE, sendo a primeira modificada com polibutadieno e a segunda composta por zircônia pura. As colunas baseadas em carbono grafitizado são comercializadas pela Thermo Scientific ${ }^{[20]}$ com o nome de Hypercarb e possuem dimensões de UHPLC, porém são recheadas com partículas de $3 \mu \mathrm{m}$.

\subsection{Instrumentação}

Em termos de instrumentação, a UHPLC pode ser considerada uma técnica bem estabelecida, uma vez que é comercializada por praticamentetodosos grandesfabricantes de equipamentos de cromatografia líquida (Waters - ACQUITY UPLC, Agilent - 1290 Infinity LC System, Thermo Scientific Dionex - UltiMate 3000 RSLC, Shimadzu - Nexera UHPLC, Thermo Scientific - Accela High Speed LC, JASCO - X-LC, Perkin-Elmer - Flexar UHPLC, Knauer - PLATINblue UHPLC e Hitachi - LaChromUltra), permitindo, dessa forma, a expansão e o emprego da UHPLC nas análises de rotina de um laboratório.

As modificações requeridas para que um equipamento de UHPLC possa ser empregado com alto desempenho cromatográfico vão além da capacidade de trabalhar a elevadas pressões $^{[1,4,5,13]}$. É necessário também possuir um sistema de bombeamento robusto, um sistema de injeção rápido, exato e preciso na faixa de 
pequenos volumes, volumes internos reduzidos (conexões, alça de amostragem, cela do detector e bombas), detectores com alta taxa de aquisição de dados e colunas e FE apropriadas.

Atualmente, os novos desenvolvimentos e/ ou as melhorias relacionadas aos equipamentos de UHPLC estão direcionados, principalmente, à busca pela diminuição da dispersão da banda cromatográfica, como forma de alcançar a máxima eficiência das colunas com dimensões de UHPLC, sendo necessária, desta forma, a redução do volume extracoluna e também desenvolver sistemas de injeção mais rápidos. Além disso, vem sendo expandida a faixa de temperatura do forno de coluna e também a pressão máxima na qual o equipamento é capaz de operar.

Outro fator importante que impulsionou a expansão da UHPLC foi o desenvolvimento de detectores capazes de adquirir e processar os dados suficientemente rápido para serem compatíveis com os picos estreitos resultantes destas análises cromatográficas ${ }^{[1,4,5,8,21]}$. Desde a introdução da técnica, detectores ópticos, UV-VIS e por arranjo de diodos, com as modificações necessárias, volume da cela do detector reduzido e longo caminho óptico, foram disponibilizados e comercializados para serem empregados nas determinações por UHPLC. Atualmente, o acoplamento da UHPLC com a espectrometria de massas também já está consolidado, com espectrômetros de massas com capacidade de trabalhar com baixos tempos de residência ( $d$ well time), baixos tempos de troca de canal (inter-channel delay) e baixos tempos de troca de varredura (inter-scan delay), de tal forma que forneçam uma quantidade de pontos suficientes para a construção do pico cromatográfico, permitindo que as mais diversas áreas de aplicação tenham acesso ao emprego da UHPLC.

\section{Desafios atuais para o emprego da UHPLC $\times$ HPLC}

Além da necessidade de instrumentos específicos (UHPLC) para o emprego eficiente das partículas $\leq 2 \mu \mathrm{m}$ e do custo dos equipamentos e dos consumíveis serem superiores aos de HPLC, os principais desafios estão relacionados com o uso contínuo do equipamento e estão descritos a seguir.

\subsection{Efeitos decorrentes da redução das tubulações e da porosidade dos filtros da coluna}

Comparando-se um sistema de UHPLC com um sistema de HPLC percebe-se uma redução significativa nos diâmetros internos (d.i.) das tubulações, que possuem tipicamente d.i. de 0,175-0,125 mm em um sistema de HPLC para d.i. de 0,125-0,0625 mm em um sistema de UHPLC e, também uma redução significativa na porosidade dos filtros que retêm as partículas da FE dentro das colunas, que é tipicamente de 2,0 ou $0,5 \mu \mathrm{m}$ para partículas de 5 ou $3 \mu \mathrm{m}$, respectivamente, para filtros com porosidade de $0,2 \mu \mathrm{m}$ para partículas $\leq 2 \mu \mathrm{m}$. A redução dos d.i. das tubulações é necessária para diminuir o volume extracoluna e, consequentemente, evitar o alargamento do pico cromatográfico, enquanto que a diminuição da porosidade dos filtros é necessária para reter as partículas de $\mathrm{FE} \leq 2 \mu \mathrm{m}$ no interior da coluna, mantendo um leito cromatográfico estável. Porém, estas alterações fazem com que as tubulações e os filtros da coluna de um sistema de UHPLC sejam muito mais suscetíveis ao entupimento que aquelas empregadas em HPLC.

Em vista disso, para evitar a entrada de qualquer tipo de material particulado, o uso rotineiro do UHPLC requer cuidados com a limpeza cromatográfica bem mais rigorosos que aqueles empregados em um sistema de HPLC, e 
envolvem $^{[22,23]}$ : (i) a amostra a ser injetada, deve ser filtrada com filtros de porosidade de $0,2 \mu \mathrm{m}$, mesmo se ela já tiver sido centrifugada, um procedimento comumente empregado, de forma satisfatória, para as amostras a serem injetadas em um sistema de HPLC. Além disso, é recomendado que seja empregado um filtro de linha com porosidade de $0,2 \mu \mathrm{m}$, como precaução; (ii) a fase móvel, deve ser filtrada com filtros de porosidade de $0,2 \mu \mathrm{m}$ (que são capazes de remover as bactérias presentes na solução) e, além disso, as FM aquosas, incluindo as tamponadas, devem ser substituídas diariamente, uma vez que pode ocorrer um crescimento microbiano que é capaz de bloquear as conexões, e o reservatório da FM deve ser substituído por um limpo cada vez que a FM for reabastecida, para evitar que a contaminação microbiana presente no frasco se transfira para a nova FM. Cuidados também devem ser tomados com o possível desgaste dos selos do pistão e dos rotores da válvula de injeção, que podem introduzir material particulado no sistema em decorrência do aumento da fricção, principalmente quando são empregadas pressões elevadas, danificando-os e reduzindo-lhes o tempo de vida útil.

Em vista disso, cabe ressaltar que os cuidados necessários com a "higiene cromatográfica" em um sistema de UHPLC devem ser bem mais rigorosos que aqueles empregados em um sistema de HPLC, porém, se realizados corretamente, o emprego rotineiro do UHPLC é totalmente adequado e possível.

\subsection{Efeitos decorrentes do uso de pressões elevadas}

A pressão resultante do emprego de colunas recheadas com partículas $\leq 2 \mu \mathrm{m}$ e das altas velocidades lineares de FM, à primeira vista, é apenas uma consequência para que análises mais rápidas sejam alcançadas. Entretanto, o emprego de pressões elevadas pode gerar alguns inconvenientes durante o desenvolvimento e a aplicação do método cromatográfico, e é discutido a seguir.

\subsubsection{Efeitos de alteração da pressão na coluna}

Giddings $^{[24]}$, há mais de 40 anos, apontou que a pressão poderia provocar mudanças no volume molar das moléculas. Em uma análise cromatográfica, isto pode implicar em mudanças na seletividade (alteração na ordem de eluição dos compostos) ou na retenção cromatográfica (provocando o deslocamento ou a sobreposição de picos cromatográficos) $)^{[22,25,26]}$.

Este fato, porém, somente começou a despertar interesse e atenção com o desenvolvimento e a comercialização de equipamentos de UHPLC que oferecem a possibilidade de operar em pressões elevadas, nos quais as mudanças de volume molar das moléculas estão mais propensas a ocorrerem, sendo praticamente irrelevante quando se trabalha com HPLC.

Desta forma, a seletividade derivada da pressão deve ser levada em consideração quando houver a necessidade de alterar métodos existentes, principalmente quando envolver uma modificação brusca na pressão e/ou, quando um método é desenvolvido em um UHPLC para ser, posteriormente, transferido para um sistema de HPLC. Esta prática tem sido comum em indústrias químicas e farmacêuticas, que, para a redução de custos e tempo, desenvolvem novos métodos em sistemas de UHPLC, porém, muitos deles, para serem posteriormente transferidos para sistemas de HPLC e empregados rotineiramente. Entretanto, a transferência de um método desenvolvido por UHPLC para HPLC não é tão simples, principalmente quando os métodos desenvolvidos envolvem a análise simultânea de vários compostos com picos estreitos e/ou com baixa detectabilidade, em um curto tempo de 
análise, uma vez que os problemas de coeluição ou mudança de retenção dos compostos podem ocorrer.

Na Figura 1, pode ser visualizado um exemplo de como a alteração da pressão na coluna pode afetar o volume molar dos compostos e, desta forma, alterar a seletividade e a retenção cromatográfica. Nos cromatogramas apresentados, pode-se verificar que houve a inversão da ordem de eluição dos compostos 3 e 4 e 5 e 6, e um aumento significativo na retenção do composto 5, com o aumento da pressão cromatográfica de 43 para 739 bar ( 620 para 11000 psi), obtido pela introdução de um capilar restritivo na saída da coluna, sendo que as demais condições cromatográficas foram semelhantes nos dois experimentos.

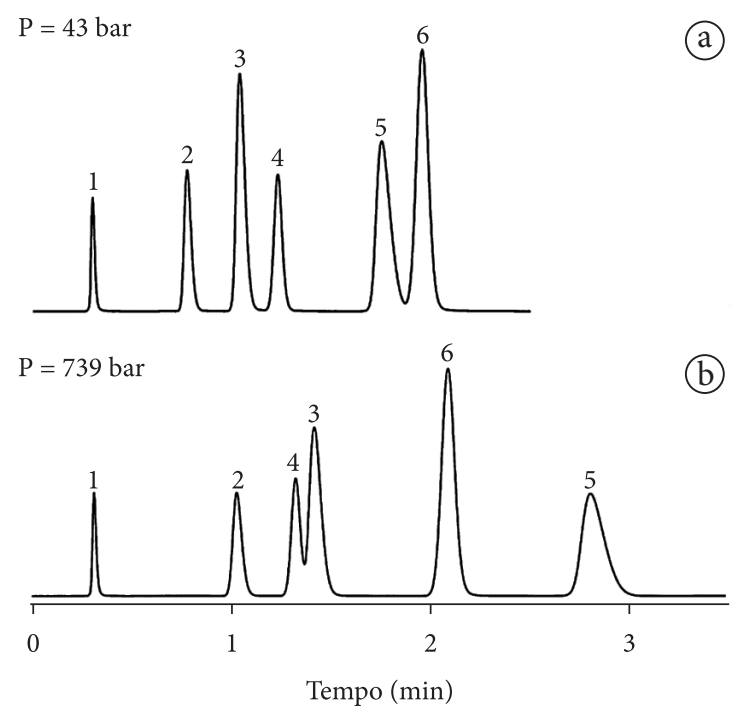

Figura 1 Cromatogramas ilustrando o efeito da pressão na seletividade e na retenção cromatográfica: (a) 43 bar e (b) 739 bar (obtida com o auxílio de um capilar restritivo de $25 \mathrm{~cm}$ ), empregando um equipamento de UHPLC e uma coluna XBridge C18 $\mathrm{BEH}(50 \mathrm{~mm} \times 2,1 \mathrm{~mm}, 5 \mu \mathrm{m})$ e como FM: acetonitrila: 0,025 mol/L fosfato de potássio $\mathrm{pH} \mathrm{2,7} \mathrm{(30:70,} \mathrm{v/v).}$ Identificação dos picos: 1 - tioureia, 2 - propranolol, 3 - difenidramina, 4 - acetofenona, 5 - protripilina e 6 - nitrobenzeno. Adaptada de Fallas et al. ${ }^{[25]}$.

\subsubsection{Efeito do aquecimento por atrito}

Quando um líquido (FM) passa através de um leito cromatográfico formado de pequenas partículas de FE, o atrito entre as duas fases provoca um aquecimento ${ }^{[22,27-30]}$. Este aquecimento é pouco significativo em se tratando de HPLC, na qual são empregadas colunas de 15-20 cm de comprimento, recheadas com partículas de FE de 3 ou $5 \mu \mathrm{m}$ e operadas a pressões de 100-200 bar ( 1400-3000 psi). Entretanto, quando são empregadas colunas de $5-10 \mathrm{~cm}$ de comprimento recheadas com partículas de $\mathrm{FE}$ $\leq 2 \mu \mathrm{m}$ e operadas a pressões de 400-1000 bar ( 6000-15000 psi), pode ser gerada uma quantidade de aquecimento que passa a ser significativa. Este calor é dissipado ao longo e através da coluna cromatográfica, resultando em gradientes de temperatura longitudinal e radial que podem influenciar na retenção e na eficiência cromatográfica e são dependentes do controle da temperatura da parede externa da coluna.

Quando a temperatura da parede externa da coluna é controlada (mantida constante), um perfil de gradiente de temperatura radial se desenvolve no interior da coluna, de tal forma que o centro da coluna torna-se mais aquecido que as laterais (Figura 2a). Esta heterogeneidade de temperatura pode provocar perda de eficiência na separação, devido ao alargamento do pico cromatográfico, uma vez que o centro da coluna estando mais aquecido faz com que as moléculas que estão no centro da banda cromatográfica se movimentem mais rapidamente que aquelas próximas da parede da coluna, resultando em um pico cromatográfico com cauda. Quando a temperatura da parede externa da coluna não é rigorosamente controlada (não é mantida constante), ocorre o desenvolvimento de um gradiente de temperatura longitudinal no interior da coluna, de tal forma que a temperatura na saída da coluna é superior a de entrada (Figura 2b). $\mathrm{O}$ gradiente de temperatura longitudinal pode 
Gradiente de temperatura radial $\mathrm{T}=$ constante

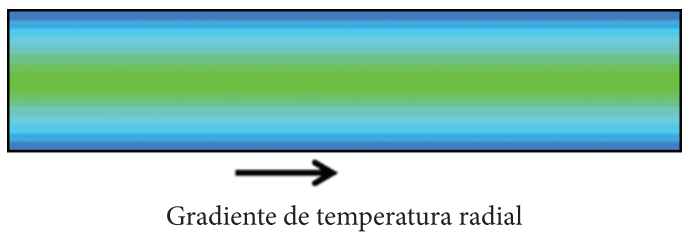

Gradiente de temperatura longitudinal $\mathrm{T}=$ não constante

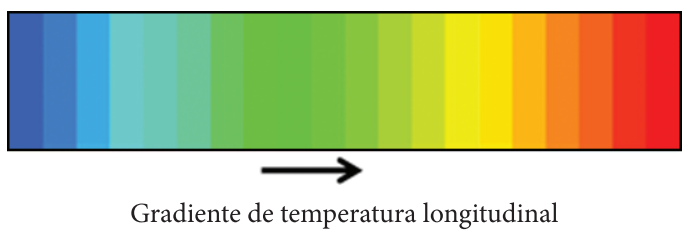

Figura 2 llustração dos gradientes de temperatura que se desenvolvem no interior da coluna resultantes do aquecimento por atrito. Adaptada de Gritti e Guiochon $^{[28]}$.

provocar alterações nos tempos de retenção dos compostos, devido às alterações da temperatura média ao longo da coluna.

Em vista disso, cabe ressaltar que é necessário considerar a possibilidade de estar ocorrendo o aquecimento por atrito quando são desenvolvidos novos métodos de separação envolvendo pressões elevadas no UHPLC, e também quando um método é transferido do HPLC para UHPLC. Alguns artifícios que podem ser empregados para reduzir esses efeitos quando um método desenvolvido por HPLC for transferido para UHPLC são: reduzir o diâmetro interno da coluna, acoplar várias colunas em série, empregar uma temperatura de trabalho em UHPLC menor que a empregada em HPLC e, se necessário, ajustar o método para que ele seja adequado para a análise por UHPLC.

\section{Considerações finais}

Diante do contexto apresentado neste artigo, pode-se concluir que a UHPLC já está bem estabelecida, tanto em termos de fases esta- cionárias como de instrumentação. É uma técnica que apresenta como vantagens a diminuição considerável no tempo de análise, a facilidade de transferência de um método desenvolvido por HPLC para UHPLC, uma grande variedade de colunas, de equipamentos e de detectores disponíveis comercialmente, indicando que, possivelmente, num futuro próximo, ela venha a superar a HPLC em análises de rotina, por também gerar menores quantidades de resíduos, atendendo aos apelos da Química Verde.

Como qualquer outra técnica, também possui as suas dificuldades ou limitações, principalmente no que diz respeito ao uso rotineiro, como a exigência de maiores cuidados com a limpeza cromatográfica e os problemas decorrentes do emprego de pressões elevadas, que podem ser superados a partir do entendimento e conhecimento dos usuários da técnica acerca deles. Entretanto, estas dificuldades não têm limitado o emprego e a expansão da UHPLC.

\section{Agradecimentos}

À FAPESP e ao CNPq pelo apoio financeiro.

\section{Referências}

1 Maldaner L, Jardim ICSF. O estado da arte da cromatografia líquida de ultra eficiência. Química Nova 2009; 32(1):214-222. http://dx.doi.org/10.1590/ S0100-40422009000100036

2 Guillarme D, Ruta J, Rudaz S, Veuthey JL. New trends in fast and high-resolution liquid chromatography: a critical comparison of existing approaches. Analytical and Bioanalytical Chemistry 2010; 397:1069-1082. PMid:19998028. http://dx.doi.org/10.1007/s00216009-3305-8

3 Natishan TK. Developments in fast liquid chromatographic analysis of pharmaceuticals. Journal of Liquid Chromatography \& Related Technologies 2011; 34:1133-1156. http://dx.doi.org/10. 1080/10826076.2011.588058 
4 Nováková L, Vlcková H. A review of current trends and advances in modern bio-analytical methods: Chromatography and sample preparation. Analytica Chimica Acta 2009; 656:8-35. PMid:19932811. http:// dx.doi.org/10.1016/j.aca.2009.10.004

5 Núñez O, Gallart-Ayala H, Martins CPB, Lucci P. New trends in fast liquid chromatography for food and environmental analysis. Journal of Chromatography A 2012; 1228:298-323. PMid:22153282. http://dx.doi. org/10.1016/j.chroma.2011.10.091

6 Wang Y, Ai F, Ng SC, Tan TTY. Sub-2 $\mu$ m porous silica materials for enhanced separation performance in liquid chromatography. Journal of Chromatography A 2012; 1228:99-109. PMid:21925665. http://dx.doi. org/10.1016/j.chroma.2011.08.085

7 Lanças FM. Aumentando a eficiência das colunas de HPLC por meio da diminuição do diâmetro das partículas da fase estacionária: até onde? Scientia Chromatographica 2011; 3(1):17-23. http://dx.doi. org/10.4322/sc.2011.002

8 Majors RE. New chromatography columns and accessories at Pittcon 2011:Part I. LCGC North America 2011; 29(3):218-235.

9 Majors RE. New chromatography columns and accessories at Pittcon 2012: Part I. LCGC North America 2012; 30(4):290-310.

10 Waters. HSS (High Strength Silica)Technology [cited 2012 jul.]. Available from: http://www.waters. com/waters/nav.htm?cid=134618105.

11 Waters. CSH (Charged Surface Hybrid) Technology [cited 2012 jul.]. Available from: http://www.waters. com/waters/nav.htm?cid=134618101.

12 Maldaner L, Collins CH, Jardim ICSF. Fases estacionárias modernas para cromatografia líquida de alta eficiência em fase reversa. Química Nova 2010; 33(7):1559-1568. http://dx.doi. org/10.1590/S0100-40422010000700024

13 Fekete S, Oláh E, Fekete J. Fast liquid chromatography:The domination of core-shell and very fine particles. Journal of Chromatography A 2012; 1228:57-71. PMid:21982449. http://dx.doi. org/10.1016/j.chroma.2011.09.050

14 Wang X, Barber WE, Long WJ. Applications of superficially porous particles: High speed, high efficiency or both? Journal of Chromatography A 2012; 1228:72-88. PMid:21855879. http://dx.doi. org/10.1016/j.chroma.2011.07.083

15 Majors RE. The increasing role of superficially porous particles in HPLC. LCGC North America 2010; 28(12):1014-1020.
16 Lanças FM. O renascimento das partículas superficialmente porosas ("core Shell particles") em HPLC. Scientia Chromatographica 2010; 2(2):47-54.

17 Santos-Neto AJ. Como obter maior eficiência com partículas superficialmente porosas em HPLC. Scientia Chromatographica 2011; 3(1):65-87. http:// dx.doi.org/10.4322/sc.2011.005

18 Borges EM, Bottoli CBG, Collins CH. Possibilidades e limitações no uso da temperatura em cromatografia líquida de fase reversa. Química Nova 2010; 33(4):945-953. http://dx.doi.org/10.1590/ S0100-40422010000400033

19 ZirChrom. ZirCrom ${ }^{\bullet}$ UHPLC Columns [cited 2012 jul.] Available from: http://www.zirchrom.com/ UHPLC.asp.

20 Thermo Fisher Scientific Inc. Hypercarb High Temperature Columns [cited 2012 jul.] Available from: https://www.thermoscientific.com/ecomm/servlet/ productsdetail_11152_L11389_81922_11963008_-1.

21 Guillarme D, Schappler J, Rudaz S, Veuthey JL. Coupling ultra-high-pressure liquid chromatography with mass spectrometry. Trends in Analytical Chemistry 2010; 29(1):15-27. http://dx.doi. org/10.1016/j.trac.2009.09.008

22 Dolan JW. UHPLC tips and techniques. LCGC North America 2010; 28(11):944-950.

23 Guillarme D, Veuthey JL. Requirements for UHPLC instruments, method development in UHPLC and method transfer from regular HPLC to UHPLC [cited 2012 jul.]. Guidelines for the use of UHPLC instruments; 2009. White Paper. Available from: http:// shop.perkinelmer.com/content/applicationnotes/ wht_guidelinesforuhplcinstruments.pdf.

24 Giddings JC. Some aspects of pressureinduced equilibrium shifts in chromatography. Separation Science 1966; 1(1):73-80. http://dx.doi. org/10.1080/01496396608049435

25 Fallas MM, Neue UD, Hadley MR, McCalley DV. Investigation of the effect of pressure on retention of small molecules using reversedphase ultra-high-pressure liquid chromatography. Journal of Chromatography A 2008; 1209:195-205. PMid:18845303. http://dx.doi.org/10.1016/j. chroma.2008.09.021

26 Fallas MM, Neue UD, Hadley MR, McCalley DV. Further investigations of the effect of pressure on retention in ultra-high-pressure liquid chromatography. Journal of Chromatography A 2010; 1217:276-284. PMid:20015498. http://dx.doi. org/10.1016/j.chroma.2009.11.041 
27 Nováková L, Veuthey, JL, Guillarme, D. Practical method transfer from high performance liquid chromatography: The importance of frictional heating. Journal of Chromatography A 2011; 1218:7971-7981. PMid:21944847. http://dx.doi.org/10.1016/j. chroma.2011.08.096

28 Gritti F, Guiochon, G. Optimization of the thermal environment of columns packed with very fine particles. Journal of Chromatography A 2009; 1216:1353-1362. PMid:19150538. http:// dx.doi.org/10.1016/j.chroma.2008.12.072
29 Wolcott RG, Dolan JW, Snyder LR, Bakalyar SR, Arnold MA, Nichols JA. Control of column temperature in reversed-phase liquid chromatography. Journal of Chromatography A 2000; 869:211-230. http://dx.doi. org/10.1016/S0021-9673(99)00894-8

30 Villiers A, Lauer H, Szucs R, Goodall S, Sandra P. Influence of frictional heating on temperature gradients in ultra-high-pressure liquid chromatography on $2.1 \mathrm{~mm}$ I.D. columns. Journal of Chromatography A 2006; 1113:84-91. PMid:16476437. http://dx.doi. org/10.1016/j.chroma.2006.01.120

Recebido: 04/09/2012

Aceito: 24/09/2012 


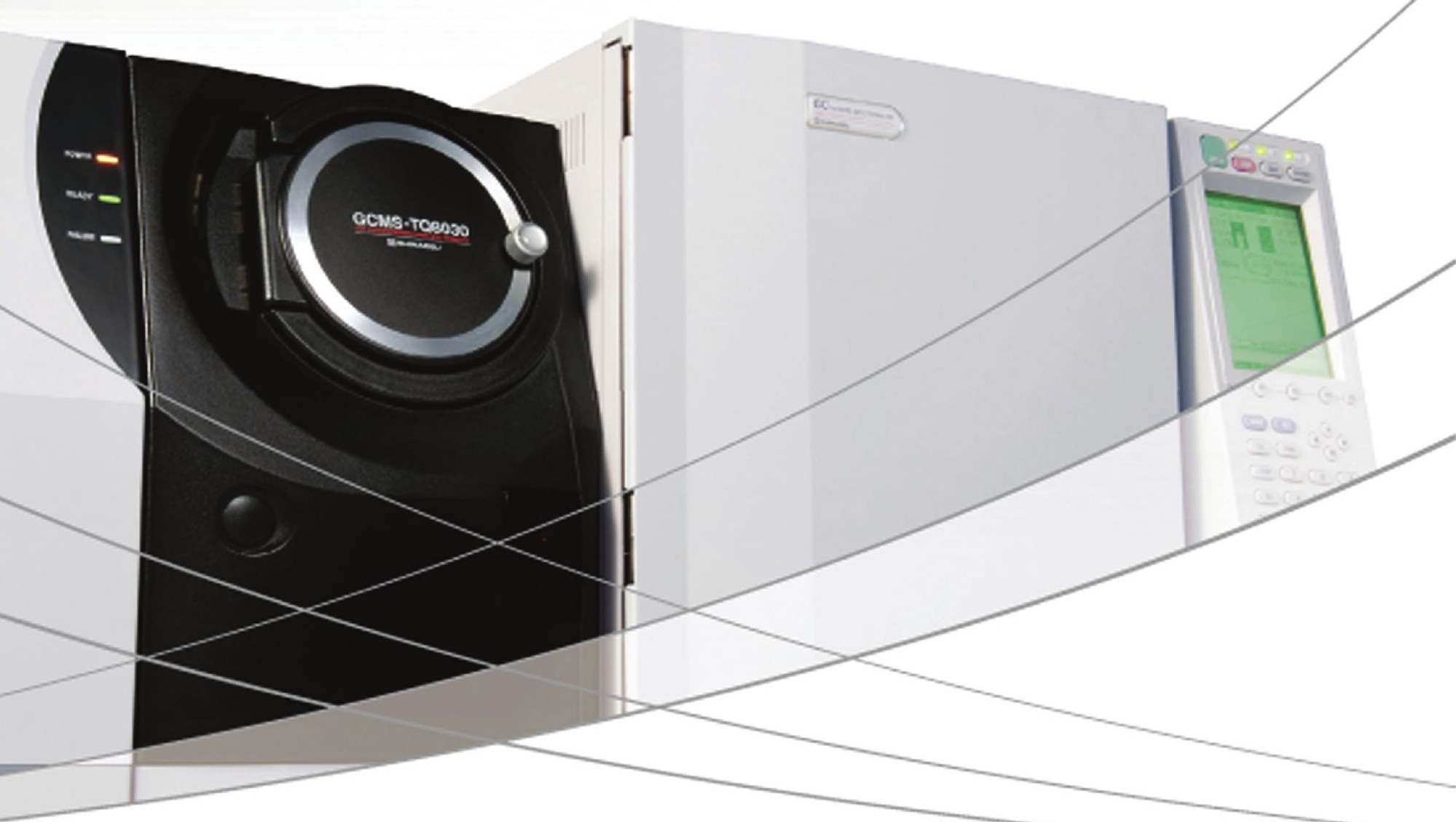

\section{Velocidade sem comparação}

A solução é o GCMS-TQ8030 Espectrômetro de Massa- Cromatógrafo Gasoso Triplo Quadrupolo, que proporciona velocidade, precisão e a operação fácil que todo cientista precisa.

\section{Alta Sensibilidade e Seletividade Reforçada}

- Patenteado pela Shimadzu, fonte de íons de alta eficiência oferece sensibilidade inigualável

- Lentes Overdrive reduzem o ruído neutro

- Variedade de modos de medição oferecem seletividade reforçada e flexibilidade de método.

\section{Desempenho de Alta Velocidade}

- UFsweeper permite 600 transições MRM por segundo - ASSP permite scanning de alta velocidade em 20.000u por segundo

- Medições Fast Scan/MRM oferecem uma riqueza de informações qualitativas e quantitativas

\section{Facilidade de Uso Definitiva}

- Função AART ajusta automaticamente a mistura e tempos de retenção MRM

- Porta de Injeção Easy sTop reduz tempo inativo em manutenção

- Câmera de fonte de lons de abertura frontal torna a manutenção mais fácil

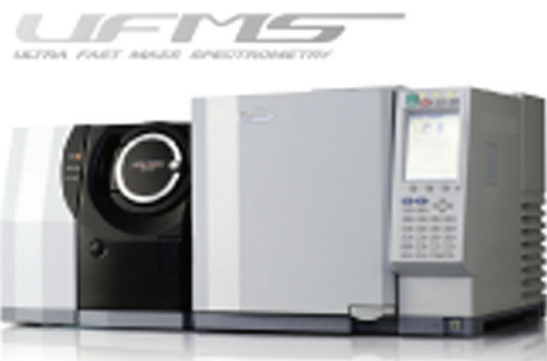

SCIENTIFIC INSTRUMENTS CO.

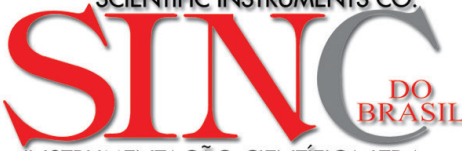

